REVIEW

\title{
New Horizons and Perspectives in the Management of Chronic Hepatitis C
}

\author{
Jaswinder Singh Sodhi, D.M., Shaheena Parveen, M.D., Riyaz-u-Saif, M.D \\ Department of Gastroenterology, Sher-i-Kashmir Institute of Medical Sciences, Soura, Srinagar
}

Hepatitis-C virus (HCV) infects 170 million people, with worldwide sero prevalence of around 3\%. ${ }^{1}$ Most of the patients develop chronic infection because the virus evades the host immune response in $55-85 \%$ of patients. Chronic HCV infection can lead to cirrhosis and hepato-cellular carcinoma. Complications of HCV-related cirrhosis are the leading indication for liver transplantation in United States and Europe.

Chronic hepatitis $\mathrm{C}$ can be cured by anti viral therapy. Currently only $40-50 \%$ of patients infected with HCVgenotype-I, treated with pegylated interferon and Ribavirin achieve a sustained virologic response (SVR) defined as absence of HCV RNA 6 months after discontinuation of treatment. SVR is associated with a durable eradication of the virus. $^{2,3}$

Till date pegylated interferon alfa and ribavirin are the standard treatment for treating chronic hepatitis C. Patients who do not respond to above treatment, the options for them are limited. Treatment is complex and difficult in patients who get hepatitis-C after liver transplantation, patients who have combined HIV and HCV infection, patients of advanced liver cirrhosis and patients of chronic hepatitis-C with genotype 1 .

HCV genotype and early virologic response (EVR) during treatment are important factors for individualization of antiviral therapy. Extended treatment duration of 72 weeks has been shown to reduce relapse rates significantly in patients who have chronic HCV genotype 1 infection and a slow virologic response compared with the standard duration of 48 weeks.

Correspondence and Reprint request to:

Dr. Jaswinder Singh

Additional Professor

Department of Gastroenterology, Sher-i-Kashmir Institute of

Medical Sciences, Soura, Srinagar-190011

Email: jassi_win@rediffmail.com
$\mathrm{HCV}$ genotype 1-infected patients with a low base line HCV RNA concentration who become HCV RNA-negative at week 4 may be treated for 24 weeks without compromising sustained virologic response rates (SVR). ${ }^{5}$

Patients who have HCV genotype 2 or 3 infection, have better response to interferon alfa than patients infected with HCV genotype 1, the standard treatment duration is 24 weeks. Smaller trials showed that shorter treatment duration of 12 to 16 weeks is equally effective as the standard treatment duration in those patients infected with HCV genotype 2 or 3 who achieve a rapid virologic response after 4 weeks of therapy.

In the Accelerate trial shorter course of therapy over 16 weeks has been shown to be as effective as a 24 weeks course in those patients who have genotype 2 or 3 infection, have a baseline viral load of $400,000 \mathrm{IU} / \mathrm{mL}$ or less, and achieve an early virologic response (EVR) at week $4 .{ }^{7}$ In patients who have genotype 2 and 3 infection without a rapid virologic response at week 4, a longer treatment duration may be necessary to optimize SVR. ${ }^{8}$

By further prolonging the treatment or increasing the dose is unlikely to improve the convenience and outcome of antiviral therapy markedly. New interferons and derivatives of ribavirin have been developed, and are currently being investigated in clinical trials.

Recent advances in structure determination of $\mathrm{HCV}$ proteins and development of a sub genomic replicon system ${ }^{9}$ besides cell culture infectious $\mathrm{HCV}$ clone, has enabled the development of a specifically targeted antiviral therapy for hepatitis C (STAT-C) ${ }^{10}$ Number of molecules are under trial which are HCV-specific inhibitors and can be effective for patients who have chronic hepatitis C.

Recent area of interest has been the exploration of how genetics influence response to treatment. Individuals of European ancestry are more likely than those of African 
ancestry to attain SVR with peginterferon and ribavirin, and genetic studies have revealed that approximately half of this difference is explained by a polymorphism near the interleukin (IL)-28B gene, which encodes interferon-gamma 3. Recent trials are underway in assessing the role of IL-28B polymorphisms in determining response to therapy with these new agents. ${ }^{11}$

\section{Newer interferons}

Albinterferon: is a long acting form of interferon alfa that results from the genetic fusion of interferon-alfa with human albumin. Albinterferon has a longer half-life and fewer side effects than pegylated interferon-alpha.

A recent clinical trial investigated antiviral efficacy and tolerability of alb interferon in combination with ribavirin in patients who had chronic hepatitis $\mathrm{C}$ genotype 1 infection and were naive to interferon-alfa-based treatment regimens. ${ }^{12}$ Overall results indicate that albinterferon plus ribavirin may offer efficacy comparable to pegylated interferon-alfa-2a plus ribavirin with half of the injections and the potential for less impairment of quality of life. The results of two pivotal phase 3 trials (Archieve 1 and 2/3) reveal that main advantage of alb interferon is lower dosing frequency of every 2 weeks. However there were more adverse effects compared to interferon especially cough alopecia and weight loss. Interstitial lung disease is a rare complication. ${ }^{13}$

Interferon-omega is a type 1 interferon that shares $70 \%$ homology of the amino-acid sequence to interferon-alfa and is derived from Chinese hamster ovary cells. Phase 1 and 2 studies have shown that interferon-omega is safe and has potent anti HCV activity. Preliminary results from a phase 2 study comparing interferon-omega $(25 \mu \mathrm{g} / \mathrm{d})$ with the combination of interferon-omega and ribavirin $(1000-1200 \mathrm{mg} / \mathrm{d})$ have shown virologic response rates 12 weeks after the end of treatment of $6 \%$ and $36 \%$ respectively. ${ }^{14}$ Interferon-omega is administered by an implantable osmotic mini pump, requiring changes every 3 months, which delivers consistent drug levels through the device outlet.

Locteron is a recombinant interferon-alfa that is released by a special biodegradable polymeric drug delivery system. The polymeric drug delivery system consists of polyester or polyether copolymers that are degraded by hydrolysis and oxidation and enables linear release of compounds. Locteron is designed to be administered every 2 weeks.

Phase 1 clinical trial revealed that a single dose of locteron was safe and well tolerated. Locteron reported fewer, less severe and shorter lasting flu-like symptoms than those subjects receiving pegylated interferon-alfa-2b.Interim results from a phase $2 \mathrm{~b}$ trial ' 480 study' demonstrated response rates comparable to peg-interferon. There was reduction in flu like symptoms. $^{15}$

Belerofon (oral interferon) is a variant of human interferon-alfa with a single amino acid replacement that is designed to lower the susceptibility of interferon-alfa to proteolytic degradation and to make it longer lasting in serum. In animal models appropriate oral doses have shown that belerofon can be absorbed from the intestine into the bloodstream and reaches blood levels comparable to those obtained by subcutaneously injected interferon-alfa. Oral belerofon is formulated as enteric-coated tablets containing the lyophilized belerofon protein. Phase 1 results on the safety, tolerability and pharmacokinetics of oral beleferon are going on.

Interferon-lambda is another new agent being evaluated for HCV. Because it uses a receptor with more limited expression, interferon- $\lambda$ may have a better safety profile than interferon-alfa. Muir and colleagues therefore performed a phase II, dose-ranging, active-control study of interferon-l plus ribavirin in treatment-naive patients with genotype 1, 2, 3, or 4 $\mathrm{HCV}$ infections. A total of 55 patients were randomly assigned to receive a single dose of interferon-lambda at doses of 80,120 , or $240 \mathrm{mg}(\mathrm{n}=45)$ or interferon-alfa at $180 \mathrm{mg}(\mathrm{n}=10)$ for pharmacokinetic analysis, followed 2 weeks later by interferon lambda or interferon-alfa plus ribavirin for 24 weeks (genotypes 2 and 3 ) or 48 weeks (genotypes1and 4). Interferon lambda was found to induce rapid viral declines with the degree of decline varying based on HCV genotype and IL-28B genotype. Clinical adverse events at or above grade 2 in severity were less frequent with interferon-lambda compared to interferon-alfa $(33 \%$ vs $50 \%)$, in particular hematologic toxicity. Grade 2 anemia (hemoglobin levels $\leqslant 10 \mathrm{~g} / \mathrm{dL}$ ) developed in $2 \%$ of patients receiving interferon-lambda versus $20 \%$ of patients receiving interferon-alfa. ${ }^{16}$

\section{Newer forms of ribavirin}

Taribavirin: It is a prodrug of ribavirin and is metabolized in liver and converted to ribavirin by hepatic adenosine deaminase. In contrast to ribavirin, taribavirin is poorly taken up by red blood cells. Two large-scale randomized phase 3 clinical trials comparing the safety and efficacy of taribavirin plus pegylated interferon-alfa-2b (VISER-1) and pegylated interferon-alfa-2a (VISER-2) versus pegylated interferon-alfa$2 \mathrm{~b} / 2 \mathrm{a}$ plus ribavirin respectively were recently completed. In both trials patients were stratified according to genotype, baseline viral load and weight. The VISER-1 trial showed a significantly lower rate of anemia among patients treated with taribavirin compared with ribavirin, however the overall rate of sustained virologic response in the VISER-1 trial was lower in taribavirn treated patients versus ribavirin treated patients $(38 \%$ versus $52 \%$. Similar results were obtained in the VISER- 2 study ( $40 \%$ versus $55 \%$ for taribavirin treated versus ribavirin treated patients. $\%) .^{17}$

\section{Site specific antiviral therapy for hepatitis $\mathbf{C}$ virus}

Recent advances in structure determination of $\mathrm{HCV}$ proteins and development of a sub genomic replicon system ${ }^{9}$ besides cell culture infectious HCV clone ${ }^{10}$ has enabled the development of a specifically targeted antiviral therapy for 
hepatitis C (STAT-C). Numbers of molecules are under trials which are $\mathrm{HCV}$-specific inhibitors (direct acting anti virals DAA) and are promising for patients who have chronic hepatitis C.

\section{Protease inhibitors}

Telaprevir It is an inhibitor of NS3/4A serine protease. It has a longer half life of bound enzyme inhibitor complex. In clinical trials telaprivir caused reduction of viral load greater than $2 \operatorname{logs} s_{10}$. Mutations causing resistance to telaprivir were seen in the replicon system during monotherapy with telaprevir. ${ }^{18}$ Telaprivir was studied in combination with pegylated interferon alfa-2a.The results of this study demonstrated additional antiviral effects of telaprivir in combination with pegylated interferon alfa-2a without any serious adverse event. Initial anti viral response to telaprivir is due to sharp reduction in wild type virus which uncovers preexisting telaprevir resistant variants. The combination of telaprevir and pegylated interferon alfa-2a inhibited wild type and resistant variants indicating that telaprevir-resistent variants are sensitive to pegylated interferon alfa-2a.

The combination of peg interferon alfa-2a and ribavirin with telaprivir given orally three times a day for 12 weeks, followed by 12 weeks of peg interferon alfa $2 \mathrm{a}$ and ribavirin in previously untreated genotype 1 infected (PROVE 1 ) patients led to a rapid reduction in serum HCV RNA levels that prevented the emergence of resistance and resulted in an SVR rate of $61 \%$ compared with $41 \%$ in those treated with pegylated interferon and ribavirin alone for 48 weeks (standard treatment). ${ }^{19}$ In telaprivir group there was significant increase in adverse effects especially skin rash which led to discontinuation of therapy in $12 \%$ of patients. In PROVE 3 study the efficacy of telaprevir was tested in patients with previously treated genotype $1 \mathrm{HCV}$ infection. In this study, patients who did not attain SVR with peginterferon and ribavirin weretreated with telaprevir plus peginterferon and ribavirin. Treatment with this triple combination was significantly more effective than re-treating with peginterferon and ribavirin alone.

From the Phase 3 ADVANCE, ILLUMINATE, and REALIZE studies showing that combining telaprevir with pegylated interferon/ribavirin produced a higher cure rate and in less time than standard therapy alone. This improvement is most notable for hard-to-treat patients including those with $\mathrm{HCV}$ genotype 1, people with liver cirrhosis, and those who did not respond to a prior course of interferon-based therapy.

Boceprevir binds reversibly to the NS3 protease active site and has potent antiviral activity. ${ }^{20}{ }^{21}$ In a phase 1 open label combination study boceprevir was evaluated in combination with pegylated interferon alfa- $2 b$ versus either agent alone in adult patients with HCV genotype 1 and were previously non responders to pegylated interferon alfa $2 b$ based therapy and was found to effectively reduce HCV RNA levels with combination therapy. ${ }^{22,23}$ SPRINT-1 trial was a phase II trial of Boceprevir in difficult-to-treat patients with HCV genotype 1. When used in combination with peginterferon alfa $2 \mathrm{~b}$ and ribavirin bociprevir use resulted in significantly higher sustained viral response(SVR) rates in the most difficult to treat patients with genotype 1 . The other studies under trial are SPRINT-2 trial which is a double-blind study which randomly assigned adults with untreated hepatitis $\mathrm{C}$ virus, genotype 1 to one of three groups. Each group received a month of peginterferon alfa- $2 \mathrm{~b}$ and ribavirin before being randomized to one of three arms. The first arm received placebo plus peginterferon-ribavirin for 44 weeks, the second arm received boceprevir plus peginterferon-ribavirin for 24 weeks, and those with a detectable HCV RNA level between weeks 8 and 24 received placebo plus peginterferon-ribavirin for an additional 20 weeks, and the third arm received boceprevir plus peginterferon-ribavirin for 44 weeks. RESPOND-2 trial studied patients with chronic hepatitis C genotype 1 who did not have a sustained response to therapy with peginterferonribavirin therapy. All patients received a month of peginterferon alfa- $2 \mathrm{~b}$ and ribavirin before being randomized to one of three arms. The first arm received placebo plus peginterferon-ribavirin for 44 weeks. The second group received boceprevir plus peginterferon-ribavirin for 32 weeks, and those with a detectable HCV RNA level at week 8 received peginterferon-ribavirin and placebo for an another 12 weeks. The third group received boceprevir and peginterferonribavirin for 44 weeks. The results of sprint 2 and RESPOND 2 trials are pending.

Ritonavir: Is a protease inhibitor approved for treatment of HIV infection with inhibitory effects on CYP3A. In experimental models the metabolism of telaprivir and boceprevir was inhibited by ritonavir. On codosing with telaprivir or boceprevir, the blood levels of the latter were increased by 15 fold.

NS4a inhibitors (ACH-806, ACH-1095): ACH806 is an antagonist of the NS4A protein which is a cofactor of the NS3 protease. ACH 806 is a protease inhibitor and prevents the formation of replicase complex after viral protein processing, a necessary step in viral replication that occurs before copying the viral RNA genome. Recent clinical trials have shown strong antiviral effect. However renal toxicity is a limiting factor.

Polymerase inhibitors: Polymerase inhibitors include nucleoside and non nucleoside polymerase inhibitors. Nucleoside analog polymerase inhibitors are converted to triphosphates by cellular kinases and incorporated into the elongating RNA strand as chain terminators. They bind to NS5B active site while as non nucleoside analogue binds to one of at least three allosteric binding sites outside the active site. Binding to one of these allosteric site leads to conformational change in the active site and thereby inhibit polymerase activity. Nucleoside analogues are more genotype selective while as non nucleoside analogues act against multiple HCV genotypes. Nucleoside analogues are less likely to select for resistance mutations than non nucleoside inhibitors. 


\section{Nucleoside analog polymerase inhibitors}

Valopicitabine is a prodrug of a nucleoside analogue, inhibiting the HCV NS5B RNA-dependent RNA polymerase. Human polymerases are not affected by this drug. Patients infected with genotype 1 and prior non responders to interferon based antiviral treatment showed a mean reduction of 0.15 to $1.21 \log 10 \mathrm{iu} / \mathrm{ml}$ after 14 days of treatment with different doses of valopicitabine ranging from $50-800 \mathrm{mg} /$ day. Ribavirin antagonizes the in vitro anti-HCV activity of 2-Cmethylcytidine, the active metabolite of valopicitabine. ${ }^{24}$ In a multicenter Phase IIb trial, E. Lawitz and colleagues studied 173 treatment-naive patients with chronic genotype $1 \mathrm{HCV}$, HCV RNA levels of at least $5 \log _{10}$, and compensated liver disease. Participants were randomly assigned to 5 arms: Pegylated interferon alfa-2a monotherapy for 4 weeks, with $800 \mathrm{mg} /$ day oral valopicitabine added in Week 5; Peginterferon alfa-2a $+200 \mathrm{mg} /$ day valopicitabine; Peginterferon alfa-2a + $800 \mathrm{mg} /$ day valopicitabine using 3 different induction regimens. Partway through the study, due to gastrointestinal side effects, the maximum valopicitabine dose was reduced to 400 mg. No subjects received ribavirin. At a dose of $200 \mathrm{mg} /$ day, valopicitabine plus pegylated interferon markedly suppresses viremia in treatment-naive patients with HCV-1 infection, with satisfactory tolerability. In a second Phase IIb study, N. Afdhal and colleagues studied 178 genotype 1 non-responders with HCV RNA levels of at least $5 \log _{10}$ and compensated liver disease; 16\% were prior partial responders (at least a $2 \log$ reduction in HCV RNA, but not full suppression) and the remainder were "true" non-responders (null responders).Here too, participants were randomly assigned to 5 arms: $800 \mathrm{mg} /$ day valopicitabine monotherapy; Peginterferon alfa + $400 \mathrm{mg} /$ day valopicitabine; Peginterferon alfa + valopicitabine at doses escalating from 400 to $800 \mathrm{mg} /$ day; Pegasys +800 $\mathrm{mg} /$ day valopicitabine; Peginterferon alfa $+1000-1200$ $\mathrm{mg} /$ day ribavirin (standard therapy). Again, the maximum valopicitabine dose was reduced to $400 \mathrm{mg}$ partway through the study (about 40 weeks). Treatment continued for 9 months after patients achieved undetectable HCV RNA, up to a maximum of 72 weeks. The valopicitabine monotherapy arm was discontinued early due to lack of efficacy. The results of this trial were less favorable than those in the treatment-naive study described above, demonstrating the difficulty of suppressing HCV in prior non-responders. Since overall sustained response rates were low, the investigators suggested that effective treatment of non-responders "will likely require the contribution of 2 or more novel and complementary agents for optimal clinical outcomes.",25,26

R1479 and R1626: These are potent inhibitors of NS5Bdependent RNA synthesis and hepatitis $\mathrm{C}$ virus replication in cell culture.R1626 is a prodrug of R1479.A phase 2 trial evaluates safety and efficacy of R1626 in combination with peginterferon alfa-2a and ribavirin. ${ }^{27}$

PSI-6130 and R7182 is a nucleoside type polymerase inhibitor is an oral cytidine nucleoside analogue. In preclinical studies no toxicity was observed. PSI-6130 was active and additive to the activity of interferon alone in these preclinical assays. A phase 1 trial of R7128 combined with peginterferon and ribavirin in genotype 1 treatment naïve patients yielded $85 \%$ rate of undetectable HCV RNA following 4 weeks of treatment compared to $10 \%$ rate in patients receiving peginterferon and ribavirin alone.

\section{Non nucleoside polymerase inhibitors}

The mechanism of action of non nucleoside polymerase inhibitors is different from those of nucleoside polymerase inhibitors. Therefore cross resistance between these two classes of drugs is unlikely to occur. They target different sites within thumb domain of the polymerase.

$\mathrm{HCV}-796$ is a non nucleoside polymerase inhibitor of the NS5B RNA-dependent RNA- polymerase that has potent antiviral activity Monotherapy showed a maximum antiviral effect after 4 days of treatment with a mean reduction of $\mathrm{HCV}$ RNA of $1.4 \log 10 \mathrm{IU} / \mathrm{mL}^{28}$

In a consecutive phase 2 study evaluating HCV-796 in combination with pegylated interferon and ribavirin clinically significant liver toxicity led to withdrawal of drug for further trials.

GS-9190 is another non nucleoside polymerase inhibitor with potent antiviral activity. The antiviral activity of GS-9190 is higher in HCV genotype 1 compared with HCV genotype 2. GS-9190 is currently being investigated in phase 1 clinical trials.

$B I L B 1941$ is an orally reversible non nucleoside inhibitor of the RNA dependent RNA-polymerase of the hepatitis C virus. The compound exhibits potent and specific inhibition of the HCV RNA-dependent RNA- polymerase in enzymatic and cell based assays. In a phase 1 trial BILB 1941 was given as monotherapy in a liquid formulation for 5 days and demonstrated significant antiviral activity in patients infected with $\mathrm{HCV}$ genotype $1 .{ }^{29}$ Increased virologic response was limited by gastrointestinal intolerance that precluded testing at higher doses. The contribution to the gastrointestinal side effects by BILB 1941 versus the constituents of the liquid formulation remains uncertain.

NS5A antagonists (A-831 and A-689): A-831 inhibits the NS5A Protein and has good therapeutic index and is currently investigated in phase 1 clinical trials. A-681 is another antiviral agent and binds to the NS5A Target at a different site. BMS790052 is an oral NS5A inhibitor. In a double blind placebo controlled trial of a single 1, 10, or $100 \mathrm{mg}$ dose of BMS- 790052 in 18 subjects with genotype 1 infection. A single $100 \mathrm{mg}$ dose resulted in decline in HCVRNA of $3.6 \log (10) \mathrm{iu} / \mathrm{ml}$.

Cyclophilin inhibitors (DEBIO-25, NIM811): Cyclophilins (Cyps) constitute one of the three families of peptidyl prolyl isomerase enzymes. CypA is the prototypical member of the Cyp family and is the predominant Cyp expressed in human cells. Recent studies indicate that CypA has an essential role in 
supporting HCV-specific RNA replication and protein expression. CypA interacts with several virally expressed proteins, including the non-structural (NS) proteins NS2, NS5A and NS5B, and may regulate diverse activities ranging from polypeptide processing to viral assembly. Cyclophilins are ubiquitous proteins in human cells that are involved in protein folding. Cyclophilin B binds to the HCV NS5B polymerase and stimulates its RNA binding capacity. The cyclophilin inhibitor DEBIO-25 has strong antiviral activity against HCV and HIV in a phase 1 trial with HCV/HIV co infected patients. ${ }^{30}$

Alispasivir (Debio-025) is a synthetic cyclosporin analogue that inhibits the binding of the host cell protein cyclophillin A to the Ns5A component of the HCV replication complex. As a result alisporivir does not induce viral resistance and is pangenotypic in its ability to supress HCV RNA replication. A phase 2 trail with Peg Interferon alfa 2a plus alisporivir $20 \mathrm{mg}$ daily revealed $4.8 \log _{10}$ decline from baseline HCV-RNA.

\section{Alfa-glucosidase 1 inhibitor}

Celgosivir: It is a new class of antiviral drug for treatment of patients of chronic hepatitis $\mathrm{C}$ The active metabolite of celgosivir is castanospermine which is a potent inhibitor of the alpha glucosidase 1 which is a host enzyme required for viral assembly and release. A phase 2 clinical trial in patients of chronic hepatitis $\mathrm{C}$ with genotype 1 with previous non response to interferon based antiviral treatment showed a 1.2 $\log 10$ decline of viral HCV RNA after treatment with celgosivir. $^{31}$

\section{Nitazoxanide}

This is an antiprotozoal drug which has shown activity against both HBV and HCV infection. The activity against $\mathrm{HCV}$ is due to phosphorylation of host protein kinases $\mathrm{R}$ and interferon alpha. In stealth C-1 trial 96 patients with genotype 4 were randomly assigned to three groups (1) Standard of care peginterferon/ribavirin for 48 weeks; Nitazoxanide monotherapy for 12 weeks followed by either (2) nitazoxanide/peginterferon for an additional 36 weeks or (3) nitazoxanide/peginterferon/ribavirin for an additional 36 weeks. SVR rates were $79 \%$ in the triple therapy arm, $61 \%$ in the nitazoxanide/pegiterferon arm and $50 \%$ in the standard of care arm. Adverse effects were similar in all the three groups except anemia which was more in ribavirin group. Stealth-2 and 3 phase-11 trials in genotype 1 patients with prior non responders and treatment naïve patients respectively are under trial. Both trials are comparing a four-week nitazoxanide lead in followed by 48 weeks of peginterferon/ribavirin/nitazoxanide with a four week placebo lead in followed by 48 weeks of peginterferon/ribavirin/pacebo. ${ }^{32}$

\section{Silybum marianum(milk thistle)}

The extract of milk thistle is silymarin and contains number of flavinoides and the major compound is silibinin. Recent studies using standardized silymarine and silibinin have shown antiviral activity against HCV. Intravenous silibinin infusions into prior non responders to peg interferon/ribavirin from 7-14 days as lead in therapy to peginterferon/ribavirin therapy plus oral silymarin revealed a significant fall in $\mathrm{HCV}$ RNA. A phase 2 randomized trials are going on.

HCV Vaccines: There is lot of diversity in different genotypes of hepatitis $\mathrm{C}$ virus and the large number of quasispecies in the infected individual leads to viral escape mutants leading to difficulties in development of effective vaccine Therapeutic vaccination involves boosting the immune response in the already infected individual while as preventive vaccination helps in preventing establishment of chronic infection in exposed individual. A hepatitis $\mathrm{C}$ immunoglobulin is being studied in phase 2 trial to evaluate its effect on recurrent hepatitis $\mathrm{C}$ following liver transplantation. A therapeutic HCV vaccine is a DNA based vaccine in which a gene encoding the HCV NS3/4A protein is introduced into the patient's skeletal muscle by a technique called DNA electroporation with the aim to stimulate $\mathrm{HCV}$-specific immune responses.

A therapeutic T-cell vaccine, based on novel adenoviral vectors was used on a small population of treatment naive patients with chronic genotype $1 \mathrm{HCV}$ infection. Intramuscular vaccination was administered 2 or 14 weeks into a 48week course of treatment with Peg-IFNa2a/ribavirin. $50 \%$ of vaccinated patients had CD4+ and CD8+ HCV specific T-cell responses at 2-8 weeks post boost, showing a strong immunogenicity for the vaccine.

Prophylactic vaccine based on novel adenoviral vectors technology (replicative-defective human Ad6 and a novel simian AdCh3 vector that encode 1985 amino-acids derived from the NS3-5 region of a genotype-1b strain). 27 healthy volunteers were vaccinated following a double prime, heterologous boost strategy. The vaccine induced polyfunctional CD4+ and CD8+ T cells responses which were maintained up to 52 weeks post prime. Overall vaccination was very well tolerated with mild/moderate local and systemic reactions and no serious adverse advents. 33,34 ,

\section{Summary}

Till date pegylated interferon and ribavirin is the standard of treatment of chronic hepatitis C. Newer long acting interferons may be convenient to give and have less side effects, however further modification of interferon therapy is unlikely to improve sustained virologic response.

Specific targeted antiviral therapy for $\mathrm{HCV}$ is a new perspective in the treatment of chronic hepatitis $\mathrm{C}$ especially for non responders to interferon and ribavirin. The new direct antivirals not only increase the viral response but also increase the rate of sustained virologic response after treatment. Hepatitis $\mathrm{C}$ vaccine is going to become a reality in coming years. 


\section{References}

1. Wasley A, Alter MJ. Epidemiology of hepatitis C: geographic differences and temporal trends. Semin Liver Dis 2000;20:1-16.

2. Fried MW, Shiffman ML, Reddy KR, et al. Combination of peginterferon alfa-2a (40kd) plus ribavirin in patients with chronic hepatitis C virus infection. $N$ Engl J Med 2002;347:925-82.

3. Shiffman MC, Suter F, Bacon BRetal. Peginterferon alfa 2a and ribavirin for 16 or 24 weeks in HCV genotype 2 or 3. NEnglJMed 2007;357:124-34.

4. Berg T, Von Wagner M, Nasser S, et al. Extended treatment duration for hepatitis $\mathrm{C}$ virus type I: comparing 48 versus 72 weeks of Peg Interferon Alfa 2a plus Ribavarin. Gastroenterology 2006;130(4):1086-97.

5. Zenzen S, Buti M, Ferenci P, et al. Efficacy of 24 weeks treatment with Peg Interferon Alfa 2B plus Ribavirin in Patients with chronic hepatitis $\mathrm{C}$ infected with geno-type I and low pretreatment viremia.J Hepatol 2006;44(1):97103.

5. Mangia A, Santoro R, Minerva N, et al. Peg Interferon alfa $2 b$ and Ribavirin for 12 vs 24 weeks in HCV genotype 2 or 3. NEnglJ Med 2005;352(25):2609-17.

7. Shiffman MV, Suter F, Bacon BR, et al. Peginterferon alfa$2 \mathrm{a}$ and Ribavarin for 16 or 24 weeks in HCV genotype 2 or 3. NEnglJMed 2007;357(2):124-34.

8. Zenzem S, Hult Crantz R, Bouvliere M, et al. Peg interferon alfa 2a plus Ribavirin for treatment of chronic Hepatitis $\mathrm{C}$ in previously treated patients infected with HCV genotype 2 or 3. J Hepatol 2004;40(6):493-9.

9. Lohmann V, Korner F, Koch J, et al. Replication of sub genomic hepatitis $\mathrm{C}$ virus RNAS in a hepatoma cell line. Science 1999;285(5424):110-3.

10. Lindenbach BD, Evans MJ, Syder AJ, et al. Complete replication of hepatitis $\mathrm{C}$ virus in cell culture. Science 2005;309(5734):623-26.

11. Alexander JT,Andrew J,Mark S, et al. Interleukin -28B Polymorphism improves viral kinetics and is the strongest pretreatment predictor of sustained virologic response in genotype 1 hepatitis $\mathrm{C}$ virus. Gastroenterology 2010;139:120-129.

12. Zenzem S, Benhamon $Y$, Bain V, et al. Antiviral response at week 12 following completion of treatment with alb interferon alfa-2b plus Ribavirin in genotype I 1FNnaïve, Chronic hepatitis C patients. J Hepatol 2007; 46:293.

13. David R Nelson, Yves Benhamoo, Wan-Long chuang et al. Albinterferon alfa-2b was not inferior to pegylated interferon-lambda in a randomized trial of patients with chronic hepatitis $\mathrm{C}$ virus genotype 2 or 3 . Gastroenterology 2010;139(4):1267-1276.

14. Navozhenov V,Zakharova N, Vino gradova E, et al. Phase
2 study of omega interferon alone or in combination with Ribavirin in subjects with chronic hepatitis C genotype I infection.J Hepatol 2007; 46:8.

15. Kraster Z, Kotzer I, Tchernev K, et al. Randomised open label,12 week comparison of controlled release interferon alfa $2 \mathrm{~b}$ plus ribavirin versus pegylated interferon alfa $2 b$ plus ribavirin in the treatment-naïve genotype 1 hepatitis C:4. Week Results 2010;139(4): 1267-1276.

16. Pegylated Interferon Lambda (PEG-IFN-Lambda) Phase II Dose-Ranging, Active-Controlled Study in Combination with Ribavirin (RBV) for TreatmentNaïve HCV Patients (Genotypes 1, 2, 3, or 4): Safety, Viral Response, and Impact of IL-28B Host Genotype through Week 12. AASLD 2010, abstract 821.

17. Benhamon Y, Pockros P, Radriguez-Torres H, et al. The safety and efficacy of Viramidine plus peginterferon alfa $2 b$ versus Ribavirin plus Peg interferon alfa-2b in therapy naïve patients infected with HCV. Phase 3 results (viserI).J Hepatol 2006;44:273 .

18. Serrazin C, Kieffer TL, Bartels D, et al. Dynamic hepatitis $\mathrm{C}$ virus genotype and phenotypic changes in patients treated with the protease inhibitor telaprevir. GE 2007; 132(5):1767-77.

19. Forestier N, Reesink HW, Weegink CJ, et al. Antiviral activity of Telaprevir (Vx-950) and Peg-interferon alfa 2a in patients with hepatitis C. Hepatology 2007;46(3):640-8.

20. Malcolm BA, Liu R, Lahser F, et al. SCH 503034, a mechanism based inhibitor of hepatitis C virus NS3 protease,suppresses polyprotien maturation and enhances the antiviral activity of alfa interferon in replicon cells. Antimicrob Agents Chemother 2006;50:101320.

21. Njoroge FG, Chen KX, Shihay et al. Challenges in modern drug discovery, a case study of boceprevir, an $\mathrm{HCV}$ protease inhibitor for the treatment of hepatitis $\mathrm{C}$ virus infection. Acc Chem Res 2008;41:50-59.

22. Sarrazin C, Rouzier R, Wagner F, et al. SCH 503034, a novel hepatitis $\mathrm{C}$ virus protease inhibitor, plus pegylated interferon alfa $2 \mathrm{~b}$ for genotype 1 non responders. Gastroenterology 2007;132:1270-8.

23. Degertekin B, Lok AS et al. update on viral hepatitis. Curr Opin Gastroenterol 2007;24:306-11.

24. Coelment L, Paeshuyse J, Windisch MP, et al. Ribavirin antagonises the invitro antihepatitis $\mathrm{C}$ virus activity of 2C-methyl cytidine, the active component of valopicitabine. Artimicrob Agents Chemother 2006;50(10); 3444-6.

25. E Lawitz, T Nguyen, Z Younes, et al. Clearance of HCV RNA with valopicitabine (NM283) plus peg-interferon in treatment-naive patients with HCV-1 infection: results at 24 and 48 weeks. (42nd EASL). Barcelona, Spain. April11-15, 2007.

26. N Afdhal, C OBrien, E Godofsky, et al. Valopictibaine 
alone or with peg-interferon, compared to peg interferon/ribavirin (PEGIFN/RBV) retreatment in patients with HCV-1 infection and prior non-response to PEGIFN/RBV: one-year results.J Hepatol 2007;46:85.

27. Roberts G, Cooksley G, Dore G, et al. Results from a phase B, multiple dose study of $\mathrm{R}$ 1626, a novel nucleoside analogue targeting $\mathrm{HCV}$ polymerase in chronic HCV genotype I patients. Hepatology 2006; 44:692.

28. Villano S, Raible D, Harper D, et al. Anti viral activity of non nucleoside polymerase inhibitor, HCV-796, in combination with pegylated interferon alfa $2 \mathrm{~b}$ in treatment naïve patients with chronic HCV.J Hepatol 2007; 4624:24.

29. Erhardt A, Deterding K, Benhamou Y, et al. Safety, pharmacokinetics and anti viral effects of BILB 1941, a novel HCV RNA Polymerase inhibitor after 5 days of oral treatment in patients with chronic hepatitis C. Antiviral therapy 2009;14;23-32.
30. Fischer G, Gallay P, Hopkins S. Current Opin Investing Drugs 2010;11(8):911-8.

31. Kaita K, Yoshida E, Kunimoto D, et al. ph11 proof of concept study of celgosivir in combination with peg interferon alfa $2 \mathrm{~b}$ and ribavirin in chronic hepatitis $\mathrm{C}$ genotype 1 non responders patients. J Hepatol 2007;46: 56-7.

32. Rossignol JF, Elfert A, Gohary $\mathrm{YZ}$ et al. Improved virologic response in chronic hepatitis $\mathrm{C}$ genotype treated with nitazoxanide, peginterferon and ribavirin. Gastroenterology 2009;136:760-763.

33. Jacobson S, McHutchison J, Boyer T. GI-5005 therapeutic vaccine plus PEG-IFN/Ribavirin significantly improves virologic response and ALT normalization at end oftreatment and improves SVR24 compared to PEG-IFN/Ribavirin in genotype 1 chronic HCV patients.J Hepatol 2010;52:465-6.

34. Folgori A et al. A T-cell HCV vaccine eliciting effective immunity against heterologous virus challenge in chimpanzees. Nature Medicine 2006;12:190 -197. 